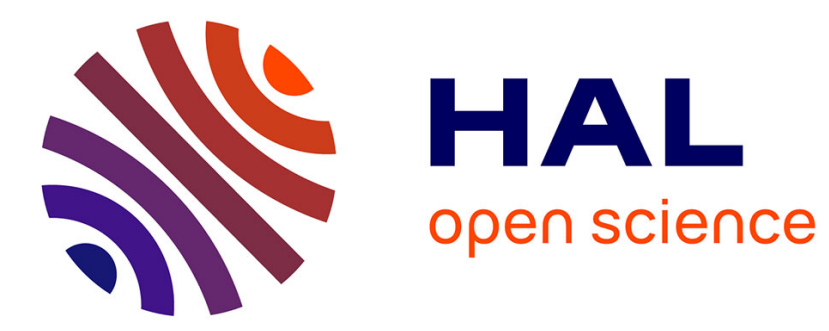

\title{
Performances obtenues avec les nouveaux magnétomètres à résonance magnétique nucléaire utilisant la polarisation dynamique par pompage électronique

\author{
R. Miquel
}

\section{To cite this version:}

R. Miquel. Performances obtenues avec les nouveaux magnétomètres à résonance magnétique nucléaire utilisant la polarisation dynamique par pompage électronique. Revue de Physique Appliquée, 1970, 5 (1), pp.135-140. 10.1051/rphysap:0197000501013500 . jpa-00243346

\section{HAL Id: jpa-00243346 https://hal.science/jpa-00243346}

Submitted on 1 Jan 1970

HAL is a multi-disciplinary open access archive for the deposit and dissemination of scientific research documents, whether they are published or not. The documents may come from teaching and research institutions in France or abroad, or from public or private research centers.
L'archive ouverte pluridisciplinaire HAL, est destinée au dépôt et à la diffusion de documents scientifiques de niveau recherche, publiés ou non, émanant des établissements d'enseignement et de recherche français ou étrangers, des laboratoires publics ou privés. 


\title{
PERFORMANGES OBTENUES AVEG LES NOUVEAUX MAGNÉTOMÈTRES A RÉSONANGE MAGNÉTIQUE NUGLÉAIRE UTILISANT LA POLARISATION DYNAMIQUE PAR POMPAGE ÉLEGTRONIQUE
}

\author{
Par R. MIQUEL, \\ Compagnie des Compteurs (C.d.G.), Gentre d'Électronique, Massy (France).
}

\begin{abstract}
Résumé. - La mesure absolue des champs magnétiques faibles (inférieure à 1 gauss) peut être effectuée en permanence et de façon très précise à l'aide d'appareils fonctionnant sur le principe de la résonance magnétique nucléaire. De tels magnétomètres utilisent comme élément détecteur un liquide riche en protons ; ils délivrent une fréquence rigoureusement proportionnelle au module du champ magnétique.

Grâce à une méthode de polarisation dynamique à double effet, il a été mis au point des transducteurs qui peuvent déceler des variations de champ aussi faibles que $10 \mathrm{~m} \gamma$ (soit $10 \mathrm{p} T$ ). La finesse de la raie de résonance et la très faible valeur d'entraînement de fréquence procurent aux appareils une grande précision. Les paramètres influant sur la précision et la sensibilité sont analysés aussi bien en ce qui concerne les capteurs eux-mêmes que les fréquencemètres qui leur sont associés.

Deux modèles de magnétomètres sont cités en exemple : l'un permet l'inscription graphique continue du champ magnétique terrestre et de ses variations avec une sensibilité de $4 \mathrm{mr} / \mathrm{mm}$; l'autre est du type numérique et, outre une lecture directe par incréments de 1,1/2 ou 1/4 de gamma, permet l'enregistrement des données sur machine imprimante ou perforatrice. Ces magnétomètres, dont la mise en service est immédiate, ne sont astreints à aucun entretien particulier.

Les performances élevées des équipements, notamment les propriétés d'isotropie et les niveaux de bruit résiduel très bas, ouvrent à ces magnétomètres un large champ d'applications en géomagnétisme et en géophysique.
\end{abstract}

Abstract. - The absolute measurement of weak magnetic fields (weaker than 1 gauss) is performed continuously and accurately by using equipments, wich operate on the principle of the Nuclear Magnetic Resonance. Such magnetometers utilize a specially-protoned liquid as sensing element. The nuclear-magnetometers generates therefore a frequency strikly proportional to the amplitude of the magnetic field.

New sensors based upon a double polarization effect have been developed, which make it possible to detect fields as small as $10 \mathrm{~m} \gamma$ (or $10 \mathrm{pT}$ ). It is because the resonance line is narrow and the frequency pulling is very small, that the equipment presents a particularly high absolute accuracy. The parameters which influence the accuracy and the sensitivity are analyzed for the sensors and the associated frequency meters.

Two types of magnetometers are presented : the first one records permanently the module of the geomagnetic field with a accuracy of $4 \mathrm{~m} \gamma / \mathrm{mm}$; the other is of a digital type and allow simultaneously for direct reading of magnetic-fields values by increments of $1,1 / 2$ or 1/4 gamma and for digital recording of the output data by printing it or punching it in a machine. The operation of such magnetometers is practically instantaneous and their maintenance does not require particular attention.

The high performances of these equipments, particularly their isotropic-qualities and very small noise levels, give them a wide range of applications in geomagnetics and geophysics studies.

Lorsque l'on veut déterminer la valeur absolue de champs magnétiques relativement faibles, c'est-à-dire dont le module est compris entre 0,2 et 1 gauss, il est maintenant indispensable de faire appel à une méthode de résonance magnétique nucléaire.

En effet, les processus mis en jeu font intervenir les propriétés corpusculaires fondamentales de la matière, qui se manifestent à notre échelle sous la forme d'une fréquence rigoureusement proportionnelle à l'amplitude du champ.

Les phénomènes utiles ne sont cependant dotés que d'une énergie extrêmement faible; aussi est-il nécessaire de les intensifier par apport indirect d'une énergie supplémentaire : nous employons dans ce but une polarisation dynamique à double effet.

La mise en œuvre d'un montage d'opposition particulier des capteurs de mesure confère à ceux-ci des qualités de faible bruit résiduel; de très petites variations de champ magnétique, de l'ordre de quelques fractions de microgauss, peuvent être ainsi mises en évidence.

Nous examinerons au cours de cet exposé les performances obtenues avec de nouveaux magnétomètres à protons destinés à fonctionner dans le champ terrestre en insistant sur les paramètres qui agissent directement sur la précision et la sensibilité de ces appareils. 
Revenons auparavant rapidement sur le principe de fonctionnement des capteurs à résonance magnétique nucléaire qui sont présentement développés ${ }^{(1)}$.

1. Principe de fonctionnement des capteurs. - Le liquide protoné, qui constitue l'élément traducteur du champ magnétique à mesurer, est soumis à une polarisation dynamique par l'intermédiaire d'un champ auxiliaire de saturation à haute fréquence.

L'accroissement de polarisation nucléaire est obtenu par couplage dipolaire entre le spin du noyau du solvant et le spin d'un électron appartenant à un radical libre dissous. La structure hyperfine du radical libre (de type nitroxyde) donne lieu, en particulier, à deux transitions distinctes : l'une correspond à un effet Overhauser direct (absorption due à une augmentation de la population du niveau quantique le plus bas), l'autre à un effet Overhauser inversé ou effet Abragam (émission due à une surpopulation du niveau le plus élevé). Les deux fréquences de résonance électronique peuvent être déterminées à l'aide de la formule de Breit et Rabi.

La même valeur de fréquence V.H.F. (appliquée à l'aide d'une cavité résonnante unique) sature simultanément la seconde transition électronique pour un demi-flacon rempli d'un liquide $A$ et la première pour un demi-flacon rempli d'un liquide B. C'est l'entraînement de fréquence produit par les solvants qui permet de saturer les deux transitions opposées à la même fréquence.

Macroscopiquement, ces polarisations inverses se traduisent sur les signaux recueillis par un angle de déphasage de $180^{\circ}$, ce qui permet le montage en opposition des deux moitiés de la bobine de mesure entourant l'échantillon. De cette façon, les influences perturbatrices extérieures (inductions statiques ou inductions fluxmétriques dues au mouvement du capteur) restent sans effet sur l'enroulement total.

Un tel montage est placé dans une boucle de réaction constituée d'un amplificateur doté d'un gain élevé et de deux demi-bobines de réinjection coaxiales aux deux demi-bobines de mesure précédentes. L'ensemble fonctionne en oscillateur nucléaire à couplage par spin (fig. 1).

Le couplage électrique est, en l'absence de champ magnétique, pratiquement nul entre le couple de bobines de réinjection et de mesure (le niveau du cou-

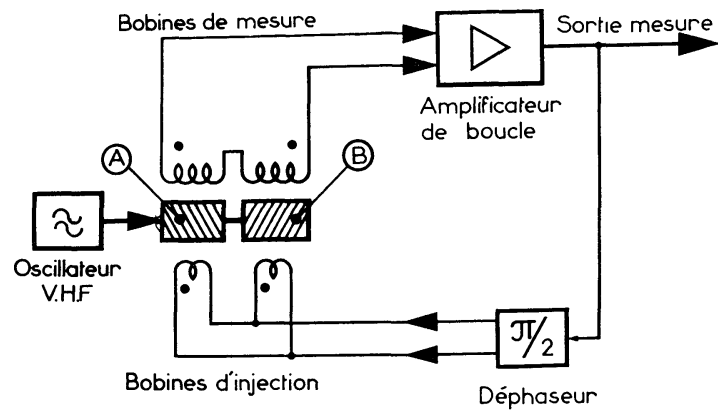

FIG. 1.

Schéma simplifié de l'oscillateur à spin nucléaire.

(1) Une description très détaillée du fonctionnement des capteurs est donnée dans la communication de A. Salvi du Centre d’Études Nucléaires de Grenoble, à laquelle on voudra bien se reporter. plage résiduel est de l'ordre de $-90 \mathrm{~dB})$. De ce fait, l'ensemble n'entre pas en oscillation. Dès qu'un champ magnétique est appliqué au capteur, l'aimantation induite des noyaux est animée d'une précession à la fréquence angulaire de LARMOR et permet le couplage des bobines : ce qui entraîne la mise en oscillation permanente du système, l'amplitude étant limitée par des effets non linéaires (régime de Van der Pol étudié par Kurochkin).

La fréquence d'oscillation, exprimée en $\mathrm{mHz}$, est proportionnelle au module du champ à mesurer, exprimé en gamma ou nanotesla $\left({ }^{2}\right)$, suivant la relation : $F=42,5759|\mathbf{H}|$; on remarque aussitôt que les faibles variations de champ conduiront à des variations de fréquence excessivement petites. Il est, par suite, nécessaire de faire appel, nous allons le voir, à des fréquencemètres particuliers.

2. Deux magnétomètres cités en exemple. - Différents types de magnétomètres ont été réalisés. Nous décrivons à titre d'exemple deux modèles, l'un de type analogique, l'autre de type numérique.

2.1. Magnétomètre D'obServatolre. - Le premier magnétomètre est plus spécialement conçu pour les observatoires géomagnétiques. Dans cette application, il permet l'enregistrement graphique permanent du champ magnétique terrestre sur un diagramme de $250 \mathrm{~mm}$ de large. Plusieurs sensibilités d'inscription sont disponibles : elles sont comprises entre $0,25 \mathrm{~mm} / \gamma$ et $250 \mathrm{~mm} / \gamma$ (c'est-à-dire $4 \gamma / \mathrm{mm}$ et $4 \mathrm{~m} \gamma / \mathrm{mm}$ ).

La valeur moyenne du champ est déterminée par changement de fréquence en comparant la fréquence issue du capteur à une fréquence de référence, dont la valeur est liée à celle du rapport gyromagnétique du proton. Ce sont les écarts par rapport à cette valeur qui sont convertis en tension continue de mesure et enregistrés.

Le fréquencemètre comporte essentiellement un oscillateur à quartz de référence associé à un analyseur de fréquences, qui délivre les valeurs discrètes de fréquences correspondant aux champs magnétiques moyens locaux $(0,25 \mathrm{G}$ à $0,7 \mathrm{G})$; un mélangeur de fréquences, auquel sont appliqués simultanément le signal de référence et le signal nucléaire à mesurer, déplace sur une fréquence centrale basse les variations de modulation de fréquence dues au champ magnétique (fig. 2).

Finalement, cette modulation de fréquence est transformée en modulation de position d'impulsions de durée étalonnée; cette durée est déterminée par le calibre de l'appareil, soit $20 \mathrm{mG}$. Les signaux ainsi obtenus qui consistent en des créneaux dissymétriques sont intégrés et la tension de sortie analogique est alors proportionnelle au champ magnétique (pour cet équipement, nous obtenons $10 \mathrm{mV}$ par gamma).

L'appareil peut être utilisé, soit pour la mesure absolue du module du champ, soit pour la mesure des variations relatives du module (emploi en variomètre). Dans ce dernier cas, la bande passante du magnétomètre est réduite à l'intervalle de $0,02 \mathrm{~Hz}$ à $0,5 \mathrm{~Hz}$. Un fonctionnement en gradiomètre est possible par l'emploi de deux capteurs séparés.

(2) On a les équivalences d'unités

$1 \gamma \Delta 10 \mu \mathrm{G} \Delta 10^{-9} \mathrm{~T}\left(\mathrm{ou} \mathrm{Wb} / \mathrm{m}^{2}\right)$

$1 \mathrm{~m} \gamma \Delta 10^{-8} \mathrm{G} \Delta 10^{-12} \mathrm{~T}$. 


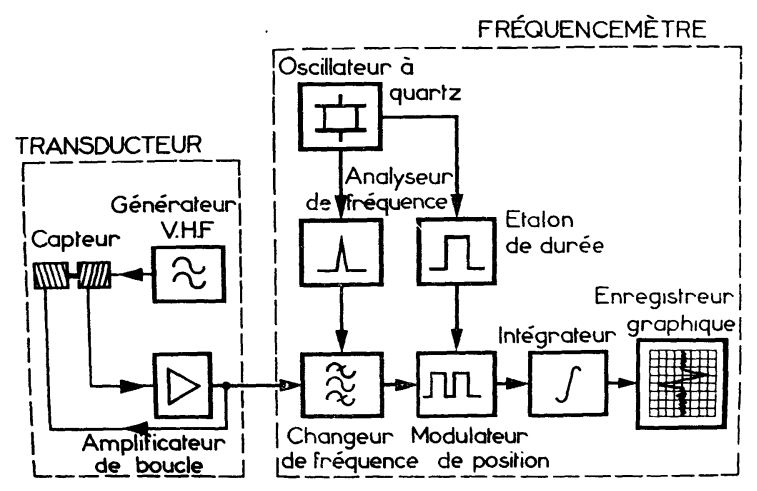

FIG. 2. - Bloc diagramme d'un magnétomètre analogique d'observatoire géomagnétique.

La dérive en température du magnétomètre en version absolue est très faible, de l'ordre de $10 \mathrm{~m} \gamma /{ }^{\circ} \mathrm{G}$ (soit $0,1 \mu \mathrm{G} /{ }^{\circ} \mathrm{G}$ ).

2.2. Magnétomètre portatif. - Le deuxième modèle de magnétomètre est du type numérique ; portatif, il trouve un emploi commode dans l'exploration géologique notamment. Le module de commande, comportant les circuits de mesure de fréquence et l'alimentation autonome, a une masse inférieure à $6 \mathrm{~kg}$.

La lecture directe de la valeur du module du champ s'effectue sur des indicateurs lumineux décimaux par incrément de $1 \gamma$.

Le principe de fonctionnement de l'appareil est le suivant : la fréquence du signal nucléaire délivré par le capteur est préalablement multipliée 16 fois par un multiplicateur de fréquence binaire apériodique; le signal à fréquence élevée est compté, c'est-à-dire intégré numériquement, pendant une durée constante de 1,5 s. La base de temps est fournie par un oscillateur à diapason qui pilote une chaîne binaire de diviseurs de fréquence (fig. 3).

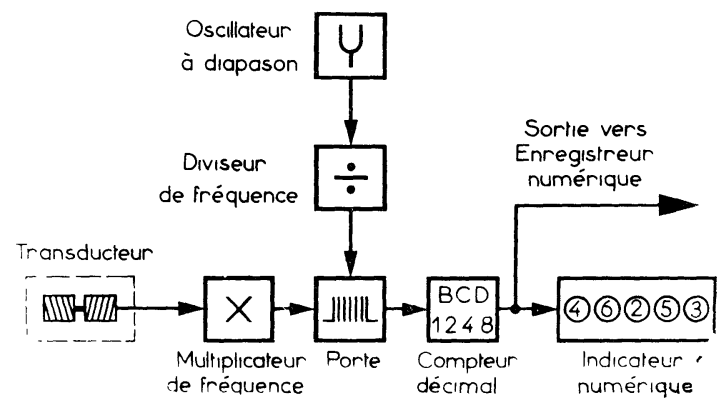

FIG. 3. - Bloc diagramme d'un magnétomètre numérique de prospection géophysique.

Une simple commutation permet d'obtenir, lorsque cela est nécessaire, des lectures au $1 / 2$ gamma et $1 / 4$ de gamma.

Ce type de magnétomètre, éventuellement à montage différentiel, peut aussi être utilisé à poste fixe. En ce cas, une sortie numérique en code BCD 1.2.4.8. est prévue : elle permet l'enregistrement des données sur machine imprimante, perforatrice ou enregistreur magnétique.

3. Performances obtenues. - De manière à bien mettre en évidence les limitations relatives aux performances des appareils, nous examinerons successive- ment les paramètres ayant une influence directe sur la précision et la sensibilité, en séparant ceux qui ont trait au capteur nucléaire et ceux qui se rapportent aux fréquencemètres électroniques.

Définissons, au préalable, le sens que nous attribuons ici aux termes de « précision » et de « sensibilité».

La précision est considérée comme la qualité globale d'un instrument de mesure; celui-ci est d'autant plus précis pour une valeur donnée de la grandeur à mesurer (ou mesurande) que les résultats qu'il donne coïncident à une plus haute approximation avec la valeur vraie du mesurande.

Nous considérerons ici la précision a priori qui résulte de l'analyse successive des erreurs élémentaires dont elle représente une synthèse. C'est plus particulièrement l'erreur ou indétermination absolue qui retiendra notre attention.

Quant à la sensibilité, elle caractérise l'aptitude d'un instrument à traduire par une large déviation (ou changement de valeur), une faible modification du mesurande.

On l'exprime au moyen du quotient du déplacement du dispositif indicateur (ou de l'accroissement de la variable observée) par la variation de la grandeur à mesurer.

Ce coefficient de sensibilité ne doit pas, rappelonsle, être confondu avec le pouvoir de résolution (la résolution représentant la plus petite variation perceptible du mesurande).

3.1. Précision du Gapteur. - Quand on désire effectuer la mesure absolue du champ magnétique, il faut envisager, outre les effets du bruit de fond qui seront analysés plus avant, quatre causes possibles d'erreur :

- le degré de connaissance du rapport gyromagnétique du proton,

- l'influence de la polarisation dynamique,

- les réactions du circuit de bouclage,

- les distorsions magnétiques dues à la construction $\mathrm{du}$ capteur.

Le rapport gyromagnétique du proton est une constante physique indépendante de tout effet extérieur; on peut considérer actuellement cette constante comme représentant un véritable étalon secondaire.

Des mesures précises ont été effectuées notamment par Bender et Driscoll en 1958 pour le National Bureau of Standards (N.B.S.) et par Yanovskii et Studentsov en 1962 pour l'Institut de Métrologie D. I. Mendéleev de Leningrad (V.N.I.I.M.). Ces auteurs ont utilisé la précession libre en eau pure désoxygénée avec une prépolarisation de l'ordre de 0,1 à $0,5 \mathrm{~T}$ (soit 1 à $5 \mathrm{kG}$ ).

Une analyse de ces mesures a été faite par Du Mond et Cohen en 1966; on peut tenir pour la plus probable (sans correction des effets d'écran diamagnétique) la valeur de $: 2,675123 \times 10^{8} \mathrm{rad} / \mathrm{T}$.s, l'erreur quadratique moyenne étant de $3 \times 10^{-6}$.

La mise en œuvre de la polarisation dynamique à double effet rend nécessaire la présence d'un radical paramagnétique dissous. Néanmoins, sa concentration est très faible, de l'ordre de $\mathrm{M} / 1000$, et la susceptibilité magnétique restant très petite, la perturbation introduite est négligeable.

Les temps de relaxation longitudinale (effet d'interaction spins-réseau) et transversale (effet d'interaction spin-spin) sont supérieurs à $2 \mathrm{~s}$ : ils justifient la finesse 
de la raie de résonance et procurent une grande précision aux magnétomètres. Pour les liquides que nous employons, la largeur de raie à mi-hauteur sur forme de Lorentz est de l'ordre de $15 \gamma$.

Le fait que l'oscillateur à couplage par spin comporte un bouclage par des bobinages de prélèvement et d'injection, on serait en droit de craindre une erreur importante due au système d'entretien. Il n'en est rien.

En effet, dans la présente méthode, c'est le produit $G Q$ (produit du gain de l'amplificateur par le coefficient de surtension de la bobine de mesure) qui intervient pour la détermination du seuil d'instabilité ( $c f$, condition de couplage critique de Townes) et non pas $Q$ seul, comme cela est le cas, généralement, dans le fonctionnement en maser par exemple.

Le gain $G$ étant très élevé, on a pu choisir un coefficient $Q$ faible vis-à-vis du coefficient de surtension du filtre à spins nucléaires et n'introduire ainsi qu'un entraînement de fréquence minime. Cette erreur reste, pour une plage de mesure de $20 \mathrm{mG}$, toujours inférieure à $0,5 \gamma$.

Notons que l'amplificateur ne doit pas présenter de rotation d'angle de phase notable dans la bande utile de fréquences. Dans nos équipements, l'erreur de phase reste limitée à $5^{\circ}$ et ne provoque un dépointage qu'à l'échelle du dixième de gamma.

Enfin, le déplacement de la raie de résonance par effet Bloch et Siegert ne conduit à une erreur relative que de $10^{-8}$, c'est-à-dire insignifiante. On sait pourtant que cet effet est proportionnel au carré du champ alternatif d'entretien créé par les bobines d'injection.

Une qualité importante des capteurs à résonance magnétique nucléaire que nous réalisons industriellement réside dans leur excellente isotropie.

Cette propriété consiste en l'invariance de la valeur de mesure d'un champ homogène, quelle que soit l'orientation du capteur dans ce champ (abstraction faite de la position " morte » correspondant à une orientation rigoureusement colinéaire au champ).

Une telle indépendance à l'orientation résulte principalement d'un amagnétisme pratiquement parfait de l'élément de mesure. La construction est effectuée avec le soin propre aux techniques spatiales, chacune des pièces élémentaires étant soumise à un contrôle rigoureux des traces magnétiques éventuelles.

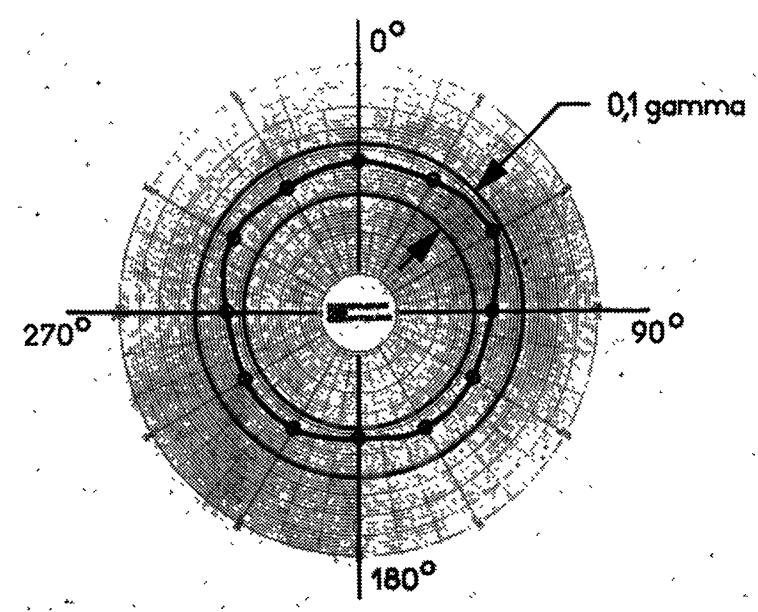

FIG. 4. - Courbe d'isotropie d'un capteur tracée en coordonnées polaires. (Contrôle d'isotropie. - Capteur no 008).
L'écart anisotropique est inférieur à $0,1 \gamma$ pour toute rotation autour de l'axe longitudinal ou suivant un axe radial quelconque du capteur. La figure 4 représente une courbe d'isotropie typique relevée en champ terrestre uniforme sur un capteur soumis à une rotation autour de son axe de symétrie. On remarquera que l'erreur reste inférieure à $0,25 \mathrm{~m} \gamma$ par degré d'angle.

Ainsi, compte tenu de tous ces effets, la précision ultime du capteur caractérisée par la valeur de l'erreur absolue globale affectant une mesure absolue de champ est bien meilleure que $1 \gamma$.

3.2. Précision du fréQuencemètre. - Cette performance sera d'autant mieux préservée que les dispositifs électroniques de mesure de la fréquence n'introduiront qu'une erreur négligeable.

Deux caractéristiques essentielles sont à prendre en considération :

- l'exactitude de la référence de fréquence,

- la linéarité de la conversion fréquence-tension, dans le cas d'une inscription analogique graphique.

Nous n'envisageons pas ici les erreurs liées à des traitements numériques, qui ne sont accessibles qu'à une échelle beaucoup plus faible. Quant aux propriétés liées au bruit de fond, elles seront examinées plus loin, en liaison avec la sensibilité.

On sait que l'avantage majeur de la résonance magnétique nucléaire est de conduire à des mesures de fréquences que l'on peut effectuer à l'aide de références très précises. Celles-ci sont constituées suivant les applications, soit par des oscillateurs à quartz, soit par des oscillateurs à diapason.

Dans le cas des quartz à régulation thermique électronique, on est assuré d'une précision à long terme supérieure au millionième. Pour nos équipements portables, des diapasons à compensation serrée permettent un calage de fréquence à peine trois fois moins précis.

Le dépouillement de diagrammes analogiques ne prend toute sa valeur que lorsque la linéarité d'échelle est rigoureuse. Le procédé qui est mis en œuvre dans les appareils d'observatoire, et que nous avons évoqué tout à l'heure, conduit à une linéarité supérieure au pour cent jusqu'au calibre de $10 \gamma$.

3.3. Sensibilité PERmise PAR LE GAPTEUR. - Les moyens actuels d'amplification électronique permettent d'obtenir des sensibilités considérables. Par exemple, il est possible - et cela est le cas du magnétomètre pour observatoire géomagnétique - d'inscrire des variations dont l'élongation graphique totale atteigne $1 \gamma$ (c'est-à-dire $10 \mu \mathrm{G}$ ) sur $250 \mathrm{~mm}$. En certains cas particuliers l'élongation totale peut même atteindre $0,2 \gamma$. Dans ces conditions, une exploitation des enregistrements ne présente un réel intérêt que si le niveau du bruit résiduel ne dépasse pas lui-même une valeur prohibitive.

La notion de rapport signal à bruit prend toute son importance, dès que l'on se préoccupe du pouvoir de résolution du magnétomètre, cette dernière propriété prenant le pas sur toutes les autres performances lorsque l'on cherche à déceler de très faibles perturbations de champ magnétique. 
Le capteur peut être envisagé comme un générateur de bruits parasites, dont les origines sont très diverses :

- bruit de partition dû aux fluctuations thermodynamiques des populations quantiques,

- bruit d'agitation thermique des éléments électriques du capteur,

- bruit d'interaction statistique entre les spins et le circuit électrique de mesure,

- bruit de l'amplificateur à semiconducteurs,

- bruit dû à une induction aléatoire par des champs extérieurs parasites.

On s'attache évidemment au premier chef à produire un signal de résonance nucléaire d'amplitude élevée. La polarisation dynamique de liquides spécialement choisis permet d'obtenir à la fois des hauteurs de raies importantes et des largeurs de raies suffisamment réduites pour que la puissance spectrale de bruit reste faible.

A cet égard, un élargissement de la raie de résonance est néfaste au maintien d'un bon rapport signal à bruit. Les gradients de champ qui ont pour effet de diminuer le temps de relaxation transversale généralisé $T_{2}^{*}$ leur sont préjudiciables. Du fait des dimensions géométriques notables du capteur, il est important que les gradients de champs extérieurs ne dépassent pas une centaine de gamma par mètre $(10 \mathrm{\gamma} / \mathrm{m}$, dans le cas de mesures précises).

La polarisation dynamique est, on le sait, appliquée à l'aide d'une cavité électromagnétique résonnante. Le mode utilisé T.E.M. (transversal électrique magnétique) a la propriété de subir l'influence vectorielle du champ magnétique. On montre que, lors d'une rotation du capteur par rapport au champ à mesurer, le volume de liquide intéressé par la polarisation peut être restreint, si la saturation n'est pas réalisée. Il est par conséquent très important de fournir à la cavité une puissance V.H.F. suffisante (dans les capteurs que nous réalisons, la puissance appliquée est de l'ordre de $700 \mathrm{~mW}$ ).

L'amplitude du signal de mesure est directement liée aux caractéristiques du bobinage de détection entourant les liquides protoniques. En particulier, le coefficient de remplissage doit être optimisé.

Nous avons vu, en début d'exposé, que le principe d'opposition des enroulements nous prémunissait contre les inductions parasites et il s'avère effectivement que le rapport signal à bruit d'amplitude du capteur nucléaire est excellent (de l'ordre de $35 \mathrm{~dB}$ ).

Si le bruit quantique (en phase) de l'oscillateur à spins peut être considéré comme négligeable à l'échelle du centième de gamma, il n'en est pas de même pour le premier étage électronique de l'amplificateur de boucle, dont le facteur de bruit constitue la limitation principale.

En particulier, l'influence de nature stochastique du bruit de fond à travers le circuit d'entretien pourrait amener, si l'on n'y prenait pas garde, une dégradation de la pureté de l'oscillateur.

Un traitement approprié du signal montre que, dans les applications géophysiques, la valeur quadratique moyenne du bruit de fond se situe aux alentours de 2/100 de gamma, lorsque la fréquence de coupure supérieure est de $0,5 \mathrm{~Hz}$.

3.4. Sensibilité liée AU fréQUencemètre. - Les résultats évoqués ici ne peuvent prendre toute leur dimension que dans la mesure où l'appareil de mesure terminal, en l'occurrence le fréquencemètre, est considéré comme parfait.

G'est le bruit de l'oscillateur électrique de référence associé à ses diviseurs binaires de fréquence qui fournit la limite principale. Pratiquement, les fluctuations erratiques de ces éléments se traduisent sur les enregistrements graphiques par un élargissement de la trace de 10 à $20 \mathrm{~m} \gamma$ crête à crête.

Ainsi, il reste raisonnable de tenir pour normalement exploitable une sensibilité d'écriture de $250 \mathrm{~mm} / \gamma$.

Les diagrammes qui sont présentés sur les figures 5 et 6 montrent un enregistrement des variations du champ magnétique terrestre effectué dans un lieu particulièrement calme, successivement avec une sensibilité de 1 , puis $0,2 \gamma$ pour toute l'étendue d'échelle.

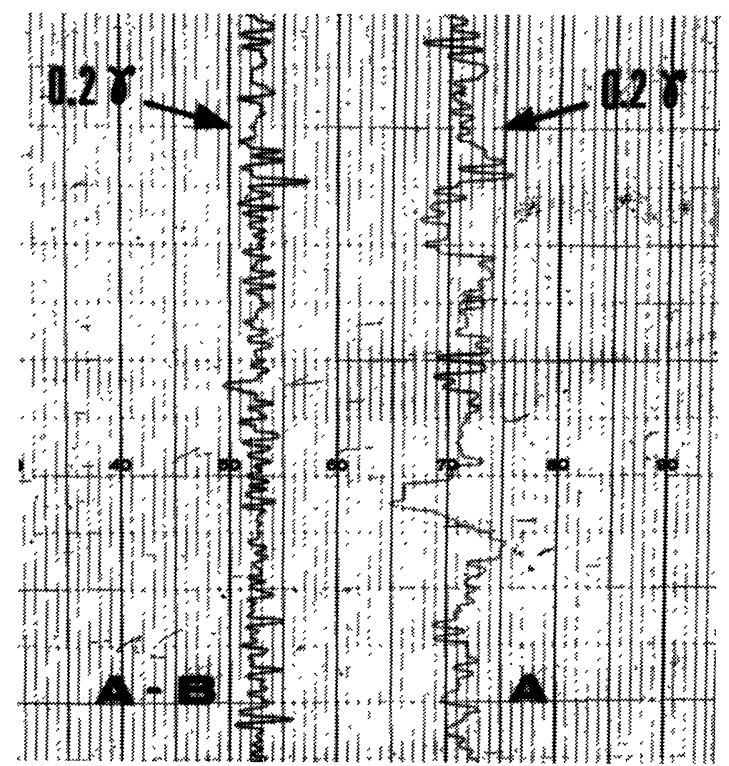

FIG. 5. - Enregistrement géomagnétique de grande sensibilité $(200 \mathrm{~m} \gamma)$.

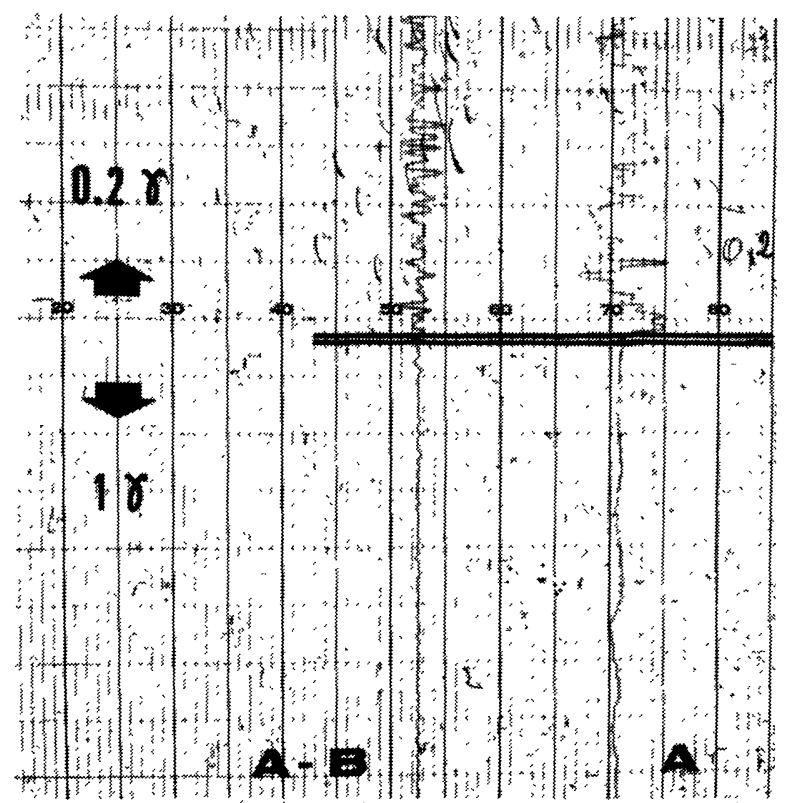

Fig. 6. - Enregistrement géomagnétique de grande sensibilité $(1 \gamma$ et $0,2 \gamma)$. 
Les durées d'enregistrement sont de l'ordre de $2 \mathrm{mn}$; la trace $\mathrm{A}$ correspond à un premier capteur, alors que la trace $\mathrm{A}-\mathrm{B}$ correspond à un relevé différentiel pris par rapport à un deuxième capteur $\mathrm{B}$, placé à distance fixe du premier.

3.5. Influence DES GONditions D'AMBiance. Bien entendu, toutes les caractéristiques que nous venons d'analyser ne peuvent être considérées comme des performances que si les équipements de mesure sont véritablement opérationnels.

Les appareils - et en particulier les capteurs à résonance nucléaire - ont été étudiés pour fonctionner dans des conditions d'environnement sévères. Notamment, leur bonne tenue aux chocs et aux vibrations en permet l'emploi sans précautions spéciales.

Les liquides nucléaires conservent leurs propriétés dans une très large étendue de températures : de $-50^{\circ} \mathrm{C}$ à $+70^{\circ} \mathrm{C}$. D'autre part, leur stabilité dans le temps est telle qu'aucun effet de vieillissement appréciable n'a pu être décelé.

Les circuits électroniques associés (oscillateur de polarisation dynamique, amplificateur de boucle, fréquencemètre) ont un fonctionnement normal entre $-20^{\circ} \mathrm{C}$ et $+50^{\circ} \mathrm{C}$.

Il est cependant une servitude d'emploi qu'il faut garder présente à l'esprit lors de mesures absolues : c'est la nécessité d'utiliser ces magnétomètres dans des champs suffisamment homogènes. Cette sujétion peut écarter, dans certains cas, leur mise en station dans des régions habitées ou des zones industrielles.

4. Conclusion. - Au terme de l'analyse que nous venons de faire des principales performances obtenues avec les nouveaux magnétomètres absolus à résonance nucléaire construits par la Compagnie des Compteurs, il ressort que ces appareils sont métrologiquement bien adaptés aux études géophysiques.

Considérés sous l'angle pratique, ils sont d'une grande commodité d'emploi, car leur mise en service est immédiate, et ils ne sont astreints à aucun entretien particulier.

Leur utilisation sous forme différentielle leur ouvre de larges possibilités dans les techniques de détection, de localisation et de prospection. En particulier, les versions en variomètres-gradiomètres permettent d'atteindre des pouvoirs de résolution suffisamment élevés pour déceler des anomalies magnétiques fines produites par des corps magnétiques très éloignés.

Ainsi les magnétomètres à protons dotés d'une polarisation dynamique à double effet constituent une nouvelle classe d'instruments de mesure fiables contribuant efficacement à l'étude des champs faibles rencontrés en géomagnétisme. 